\title{
Infrared Spectrum of the Silicon Hydride Cation $\mathrm{SiH}_{7}^{+}$
}

\author{
Yibin Cao, Jong-Ho Choi, Bernd-Michael Haas, Matthew S. Johnson, and Mitchio Okumura
}

Arthur Amos Noyes Laboratory of Chemical Physics

California Institute of Technology, Pasadena CA 91125

\begin{abstract}
Silanium ions are an important class of hypervalent molecules, and the determination of their structure will yield insights into the nature of nonclassical bonding and provide a contrast to the bonding in carbonium ions. We report the infrared spectrum of the mass-selected silicon hydride cluster ion ${ }^{28} \mathrm{SiH}_{7}{ }^{+}$, detected by vibrational predissociation spectroscopy. Silanium ions were formed in a pulsed high pressure glow discharge and cooled by the subsequent supersonic expansion. Photodissociation spectra were obtained using a tandem time-of-flight mass spectrometer: $\mathrm{SiH}_{7}^{+}$ions were mass-selected and excited by a tunable infrared laser. The resulting photofragments were detected using a reflectron as a mass analyzer. We observed a vibrational band at $3865 \mathrm{~cm}^{-1}$, which was the only one observed from $3500 \mathrm{~cm}^{-1}$ to $4200 \mathrm{~cm}^{-1}$. This result suggests that the molecule might form a symmetric complex with the structure $\mathrm{H}_{2} \cdot \mathrm{SiH}_{3}{ }^{+} \cdot \mathrm{H}_{2}$, in contrast to the species $\mathrm{CH}_{7}^{+}$, which has the structure $\mathrm{CH}_{5}^{+} \mathrm{H}_{2}$.
\end{abstract}

\section{INTRODUCTION}

Recent investigations of the chemistry of silicon hydride cations have been motivated by the possible role of ion-molecule reactions in the gas phase silane chemistry occurring during chemical vapor deposition (CVD) of silicon films, especially plasma-enhanced CVD. Both experimental ${ }^{1-9}$ and theoretical ${ }^{10-13}$ studies have $^{-1}$ focused on reactions of $\mathrm{Si}^{+}$and $\mathrm{SiH}_{3}{ }^{+}$with silane that produce hydrogenated silicon cluster ions $\mathrm{Si}_{n} \mathrm{H}_{m}{ }^{+}$. The theoretical calculations ${ }^{10-13}$ suggest that these cluster ions exhibit rather novel nonclassical bonding, analogous to but distinct from the nonclassical bonding observed in carbonium ions. ${ }^{14}$ Such bonding might even exist in monosilane cluster ions. For example, in the case of $\mathrm{SiH}_{5}{ }^{+}$, theoretical calculations suggest that an $\mathrm{H}_{2}$ is bound to $\mathrm{SiH}_{3}{ }^{+}$through a three-center two-electron bond, ${ }^{15}$ with a bond strength much weaker than that in $\mathrm{CH}_{5}{ }^{+}$.

Although the gas phase chemistry of silicon hydride ions has been studied extensively, there have been few spectroscopic studies to date. Dyke et al. ${ }^{16}$ have reported the frequency of the out-of-plane bending mode of $\mathrm{SiH}_{3}{ }^{+}$from a photoelectron spectrum of the $\mathrm{SiH}_{3}$ radical. Smith, Martineau and Davies ${ }^{17}$ have recently reported a high resolution infrared absorption spectrum of $\mathrm{SiH}_{3}{ }^{+}$cation detected in a glow discharge. $\mathrm{SiH}_{3}{ }^{+}$is the dominant ion in low pressure silane discharges, and no other species have thus far been detected by this method.

Using an ion trapping tandem mass spectrometer apparatus, Lee and co-workers have obtained infrared vibrational predissociation and multiphoton photodissociation spectra for a number of ionic clusters. ${ }^{18-20}$ This technique has proved to be a powerful method for obtaining spectra of mass-selected clusters. We have recently developed a simpler apparatus using pulsed techniques, based on the ion photodissociation spectrometer developed by Johnson and Lineberger. ${ }^{21}$ We have begun to apply the vibrational predissociation method to silicon hydride cations and in this paper we report our initial results on the infrared spectrum of the $\mathrm{SiH}_{7}^{+}$cation. 


\section{EXPERIMENTAL}

Silanium ions were generated in a pulsed high pressure discharge source which we developed recently. A mixture of $1 \%$ silane in UHP helium was mixed with UHP hydrogen using a MKS flow system to form a mixture of approximately $30 \mathrm{ppm} \mathrm{SiH}_{4}$ in $\mathrm{H}_{2}$ at 1000 torr. The gas expanded through a piezo-driven pulsed valve $^{22}$ with a pulse width of $200 \mu$ s into a $0.5 \mathrm{~mm}$ diameter channel. The gas then entered a small chamber with two tungsten electrodes $(0.5 \mathrm{~mm}$ diameter) where a high voltage pulse (typically $-1 \mathrm{kV}, 100 \mu \mathrm{s}$ wide) was applied to the anode; the timing suggested that the best conditions for ion formation occurred when the high voltage was applied during the leading edge of the gas pulse. Ions were swept out by the gas pulse through a $1 \mathrm{~mm}$ diameter, $1.5 \mathrm{~cm}$ long channel and into the first differential vacuum chamber (pumped by a 10" diffusion pump and maintained at a pressure of $2-10 \times 10^{-5}$ Torr). The plasma was cooled as the gas flowed through the channel and underwent supersonic expansion. The effectiveness of the cooling was demonstrated by the formation of cluster ions with binding energies of $3 \mathrm{kcal} / \mathrm{mol}$ or less, such as $\mathrm{H}_{3} \mathrm{O}^{+}\left(\mathrm{H}_{2}\right)$. A typical TOF mass spectrum is shown in Fig. 1. The source was operated at a repetition rate of $30 \mathrm{~Hz}$, and produced intense and stable silanium ion beams for hours during which no adjustments were needed.

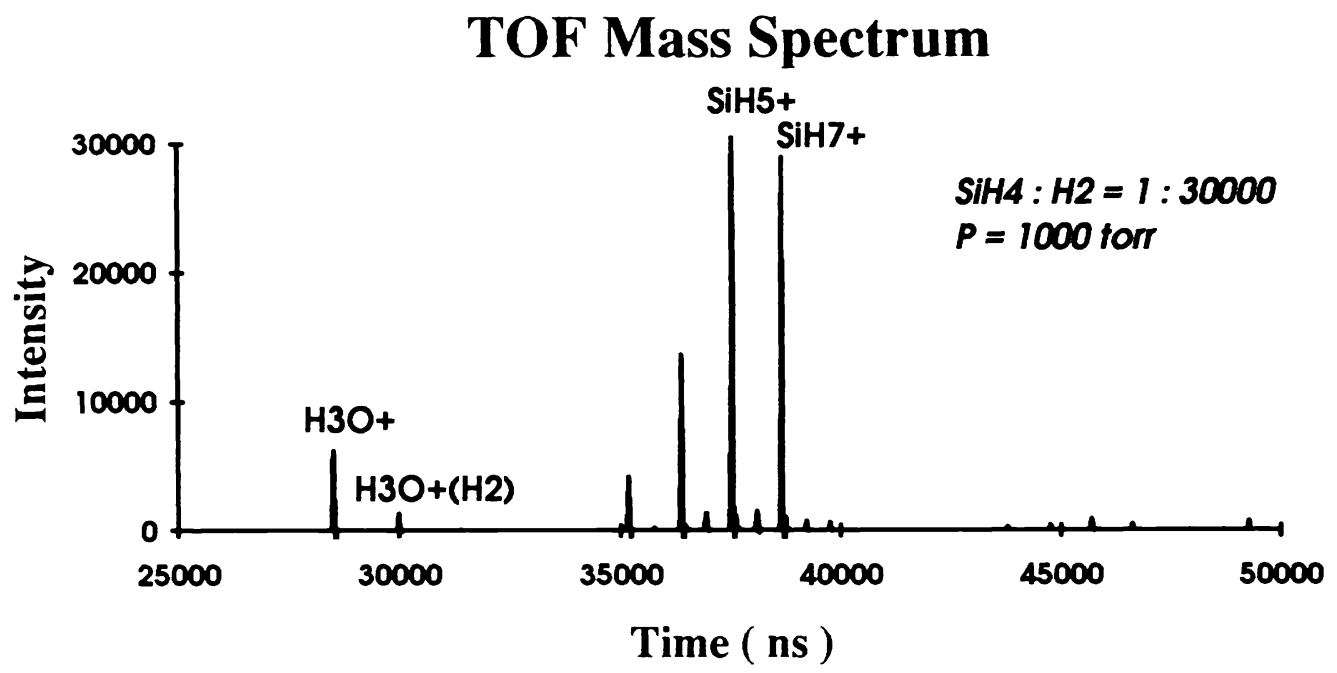

Fig. 1. Time-of-flight mass spectrum of the silanium ions. The pulsed valve stagnation pressure was 1000 Torr. The gas mixing ratio $\mathrm{SiH}_{4}: \mathrm{H}_{2}$ was $1: 30000$.

The plasma expanded supersonically and was collimated by a $3 \mathrm{~mm}$ diameter skimmer to enter a second chamber (6" diffusion pump, $5 \times 10^{-6}$ Torr) containing time-of-flight ion optics. A pulsed electric field applied between two plates $3 \mathrm{~cm}$ apart extracted the positive ions between them. The ions were further accelerated by additional electric fields and then entered a field-free region with a final kinetic energy of $1 \mathrm{keV}$. After passing through a third differential pumping region (4" diffusion pump, $1 \times 10^{-6}$ Torr) and into a photolysis/detection chamber $\left(500 \mathrm{~L} / \mathrm{s}\right.$ turbomolecular pump, $3 \times 10^{-7}$ Torr), the ions encountered a $1 \mathrm{~cm}$ long mass gate where a pulsed tranverse field was applied to deflect all incident ions except those of the mass of interest. The selected ions were focused into a packet $9 \mathrm{~mm}$ long and $5 \mathrm{~mm}$ in diameter at $1.5 \mathrm{~m}$ downstream from the acceleration optics, where they were intersected by the infrared laser beam. The ions were then mass-analyzed by a $15 \mathrm{~cm}$ long reflectron tilted at $2^{\circ}$, and detected by a microchannel plate detector. The signal was amplified and then 
collected by a LeCroy 8818 transient digitizer (100 MSamples/s) and 6010 signal averager.

The tunable, pulsed infrared radiation was produced by a $\mathrm{LiNbO}_{3}$ optical parametric oscillator (OPO). The OPO, based on the design of Brosnan and Byer, ${ }^{23}$ was pumped by a Continuum $661 \mathrm{~s} \mathrm{Nd:YAG} \mathrm{laser} \mathrm{which}$ generated $250 \mathrm{~mJ}$ pulses at $1.06 \mu \mathrm{m}$. To avoid damaging the $\mathrm{LiNbO}_{3}$ crystal, the pump beam was collimated and multipassed 14 meters to achieve near-Gaussian beam profile with a spot size of $3.5 \mathrm{~mm}\left(1 / e^{2}\right.$ diameter $)$ at the OPO entrance. The OPO was continuously tunable from $2700 \mathrm{~cm}^{-1}$ to $4300 \mathrm{~cm}^{-1}$ by simultaneously adjusting the crystal angle and the grating. Typical OPO pulse energies were 5-8 mJ with a linewidth of 1.5 $\mathrm{cm}^{-1}$. The optical path from the laser to the vacuum chamber was purged with dry air to eliminate absorption due to ambient water vapor.

A single-field reflectron was used for mass analysis of the fragment ions. Because of the negligible kinetic energy released during the vibrational predissociation, the photofragment ion (mass $m_{\mathrm{f}}$ ) had the same velocity as its parent ion (mass $m_{i}$ ). By reducing the reflectron electric field by a factor of $m_{f} / m_{i}$, the fragment ion would experience the same acceleration in the new field as the parent ion did in the original reflectron field, thus reaching the detector at exactly the parent ion arrival time in the original field. Using this procedure, the mass of the photofragment ion was determined.

Spectra were recorded by stepping the OPO wavelength and averaging the photofragment signal for 400 laser shots at each wavelength. To achieve better signal-to-noise ratio, we averaged 10 such scans. Background signal, primarily caused by collision-induced dissociation in the ion optics chamber, was subtracted by firing the laser beam alternately at the parent ion arrival time and $10 \mu \mathrm{s}$ before the ion arrival, and taking the difference. The data were then normalized with respect to the OPO laser intensity, which was measured by a Molectron joulemeter. The OPO laser wavelength was calibrated during the scan by simultaneously recording the vibrational spectrum of acetylene in a photoacoustic cell.

\section{RESULTS AND DISCUSSION}

An absorption band of $\mathrm{SiH}_{7}^{+}$was observed in the $3800-3925 \mathrm{~cm}^{-1}$ region, as shown in Fig. 2. This band has clear $P, Q$, and $R$ branches, with the $Q$ branch centered at $3865 \mathrm{~cm}^{-1}$. The only photofragment ion observed was $\mathrm{SiH}_{5}^{+}$, indicating that the photodissociation process involved was $\mathrm{SiH}_{7}{ }^{+} \rightarrow \mathrm{SiH}_{5}{ }^{+}+\mathrm{H}_{2}$. Due to the broad linewidth of the OPO laser $\left(1.5 \mathrm{~cm}^{-1}\right)$, rotational structure of this band was not resolved. There were no other absorptions found from $3500 \mathrm{~cm}^{-1}$ to $4200 \mathrm{~cm}^{-1}$.

Based on the relatively high frequency and the strength of this absorption band, we assign it to an $\mathrm{H}-\mathrm{H}$ stretching motion. This stretch is strongly perturbed, as evidenced by its $300 \mathrm{~cm}^{-1}$ frequency shift from the free $\mathrm{H}_{2}$ fundamental of $4161 \mathrm{~cm}^{-1}$. This assignment supports the notion that $\mathrm{SiH}_{7}^{+}$is an ionic cluster with at least one $\mathrm{H}_{2}$ molecule bound to the charge center. The $\mathrm{H}-\mathrm{H}$ stretch obtains its transition dipole moment through coupling with degrees of freedom that possess oscillator strength, e.g. motion of the charge center relative to the center of mass.

Since the $\mathrm{SiH}_{7}^{+}$ion was never observed before experimentally, theoretical calculations on its structure have not yet been carried out. Hu et al. have done calculations on $\mathrm{SiH}_{5}{ }^{+}$with various ab initio methods. ${ }^{15}$ Their optimized structure for $\mathrm{SiH}_{5}^{+}$is a weakly bound complex of $\mathrm{SiH}_{3}^{+}$and $\mathrm{H}_{2}$ subunits (Fig. 3a) . The best theoretical estimate of the dissociation energy $D_{0}$ for $\mathrm{SiH}_{5}{ }^{+}$is $10.3 \mathrm{kcal} / \mathrm{mol}$, less than the experimental value of $17.8 \mathrm{kcal} / \mathrm{mol}$. They also report a harmonic frequency of $3991 \mathrm{~cm}^{-1}$ for the perturbed $\mathrm{H}-\mathrm{H}$ stretch, although $a b$ initio frequencies are generally $5 \%$ to $10 \%$ higher than experimental values. 


\section{Vibrational Spectrum}

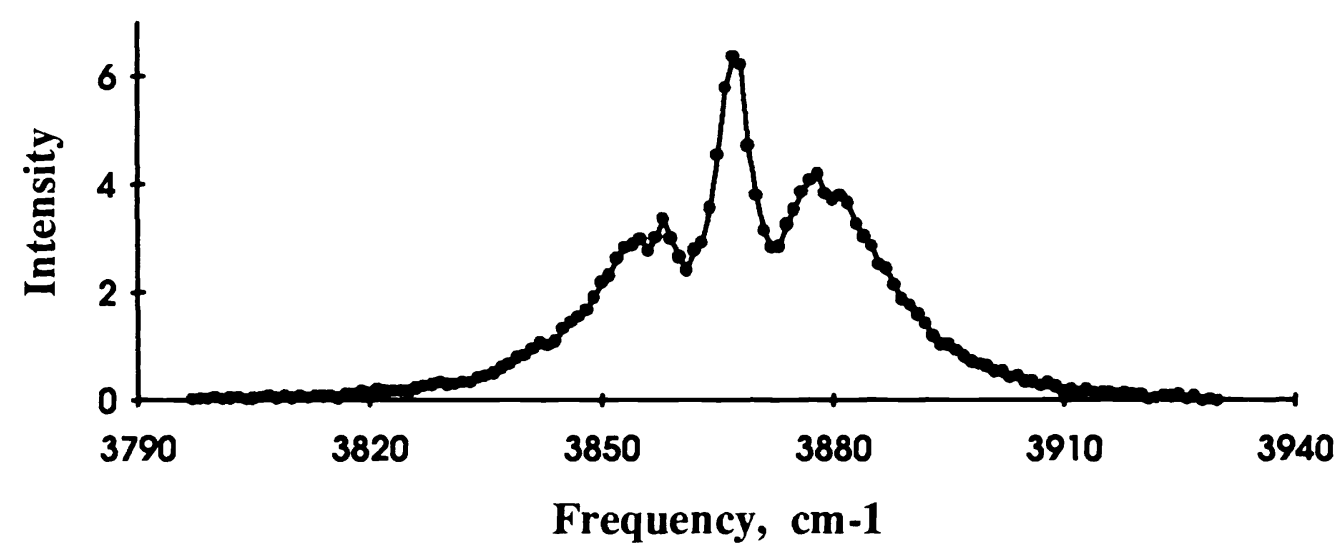

Fig. 2. Vibrational predissociation spectrum of the $\mathrm{SiH}_{7}^{+}$ion in the $3800 \mathrm{~cm}^{-1}-3925 \mathrm{~cm}^{-1}$ region. No other absorptions were found from $3500 \mathrm{~cm}^{-1}$ to $4200 \mathrm{~cm}^{-1}$.

The observed frequency of $3865 \mathrm{~cm}^{-1}$ for $\mathrm{SiH}_{7}^{+}$is close to the harmonic frequency of $3991 \mathrm{~cm}^{-1}$ for $\mathrm{SiH}_{5}{ }^{+}$predicted by $\mathrm{Hu}$ et al. This agreement at first suggests that $\mathrm{SiH}_{7}{ }^{+}$could be a complex with an $\mathrm{H}_{2}$ molecule very weakly bound to the $\mathrm{SiH}_{5}^{+}$ion core, analogous to the structure proposed by Boo, Price, and Lee for the $\mathrm{CH}_{7}^{+}$ion (Fig. 3b).$^{20}$ However, the $\mathrm{H}_{2}$ moiety would be perturbed by the $\mathrm{SiH}_{5}^{+}$core and therefore exhibit an absorption band slightly below $4161 \mathrm{~cm}^{-1}$, the vibrational frequency of a free $\mathrm{H}_{2}$. In all previous cases involving an ion-molecule cluster with $\mathrm{H}_{2}$ ligands, such an absorption band has been observed. We scanned the region from $3500 \mathrm{~cm}^{-1}$ to $4200 \mathrm{~cm}^{-1}$, but found no evidence of a second absorption band belonging to $\mathrm{SiH}_{7}^{+}$. In addition, we observed that the $\mathrm{SiH}_{7}{ }^{+}$ions could exist even under "hot" source conditions that makes mostly $\mathrm{SiH}^{+}$ ions, which suggests that the $\mathrm{SiH}_{7}^{+}$binding energy is much larger than that of $\mathrm{CH}_{7}^{+}$.

We therefore propose that $\mathrm{SiH}_{7}{ }^{+}$has the structure shown in Fig. 3c: a planar or nearly planar $\mathrm{SiH}_{3}{ }^{+}$with two $\mathrm{H}_{2}$ ligands bound to opposite faces of the ion. The two $\mathrm{H}_{2}$ ligands are approximately the same distance from the Si atom. This structure should result in two $\mathrm{H}-\mathrm{H}$ stretching bands: the symmetric and antisymmetric combinations of the two $\mathrm{H}_{2}$ stretches. The symmetric combination, which has the two $\mathrm{H}_{2}$ ligands expanding and contracting simutaneously, would induce little movement of the charge center and thus should have much weaker absorption intensity. Our current interpretation of the spectrum is that it is in fact weaker and obscured by the stronger antisymmetric mode.

Our proposed structure for $\mathrm{SiH}_{7}^{+}$is quite distinct from the structure proposed by Boo et al. for $\mathrm{CH}_{7}{ }^{+20}$ The differences can be rationalized by comparing the $\mathrm{CH}_{5}{ }^{+}$and $\mathrm{SiH}_{5}{ }^{+}$ions. The dissociation energy for $\mathrm{CH}_{5}{ }^{+} \rightarrow$ $\mathrm{CH}_{3}{ }^{+}+\mathrm{H}_{2}$ is $40 \mathrm{kcal} / \mathrm{mol}$, and the strong three-center two-electron bond in $\mathrm{CH}_{5}{ }^{+}$results in charge delocalization among the three centers. In contrast, the dissociation energy for $\mathrm{SiH}_{5}^{+} \rightarrow \mathrm{SiH}_{3}^{+}+\mathrm{H}_{2}$ is only $17.8 \mathrm{kcal} / \mathrm{mol}$. The $\mathrm{SiH}_{5}{ }^{+}$ion is thus closer to an $\mathrm{SiH}_{3}{ }^{+}\left(\mathrm{H}_{2}\right)$ complex, as $\mathrm{Hu}$ et al. predict. ${ }^{15}$ A second $\mathrm{H}_{2}$ will bind to the $\mathrm{Si}$ atom, since some of the charge remains localized there. 


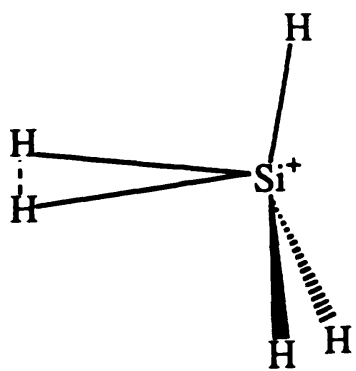

A.

\section{Molecular Structures}

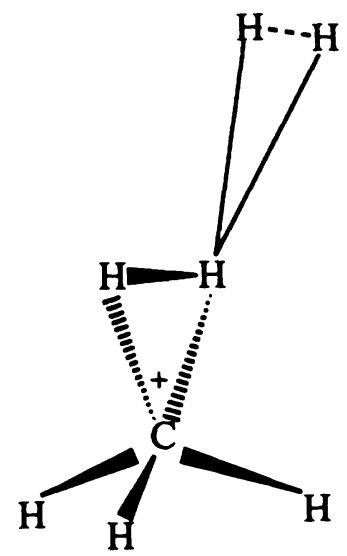

B.

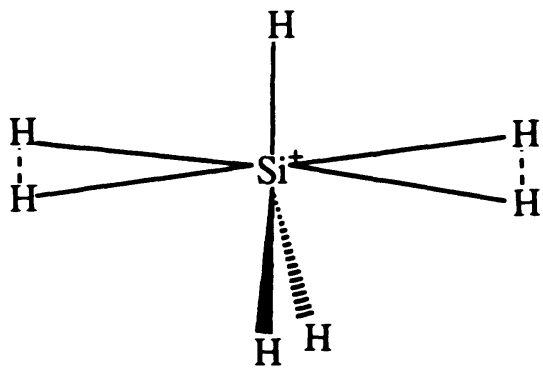

C.

Fig. 3. (a): $\mathrm{SiH}_{5}{ }^{+}$structure predicted by $\mathrm{Hu}$ et al. (b): $\mathrm{CH}_{7}{ }^{+}$structure based on the experimental results of Boo et al. (c): Our proposed structure for $\mathrm{SiH}_{7}{ }^{+}$.

These results shed some light on the structure of $\mathrm{SiH}_{5}{ }^{+}$and $\mathrm{SiH}_{7}{ }^{+}$. A more definitive structure determination requires recording the rotationally resolved spectrum. If the $\mathrm{H}_{2}$ ligands are about $2 \AA$ from the planar $\mathrm{SiH}_{3}{ }^{+}$, then $\mathrm{SiH}_{7}{ }^{+}$is a prolate top with a rotational constant $B$ of $0.85 \mathrm{~cm}^{-1}$. Our newly upgraded OPO has a linewidth of $0.15 \mathrm{~cm}^{-1}$ and will easily resolve the spectra. The next step in silanium ion spectroscopy would be $\mathrm{SiH}_{5}{ }^{+}$. Since the dissociation energy for $\mathrm{SiH}_{5}^{+} \rightarrow \mathrm{SiH}_{3}{ }^{+}+\mathrm{H}_{2}$ is $17.8 \mathrm{kcal} / \mathrm{mol}, \mathrm{SiH}_{5}{ }^{+}$spectra can be obtained through two-photon photodissociation. Larger silicon hydride ions are also believed to possess unusual geometries. In $\mathrm{Si}_{2} \mathrm{H}_{7}^{+}$, for example, the two $\mathrm{Si}$ atoms are thought to bond through an $\mathrm{H}$ atom, with a structure of $\mathrm{H}_{3} \mathrm{Si}-\mathrm{H}^{+}-\mathrm{SiH}_{3}$. By using multiphoton dissociation techniques, we hope to probe the structure and novel bonding of these fascinating species as well.

\section{ACKNOWLEDGMENT}

We gratefully acknowledge the support of a National Science Foundation Presidential Young Investigator Award CHEM-8957243 and an AT\&T Special Purpose Grant. Additional support was received from a Dreyfus Newly Appointed Faculty Award, the Irvine Foundation, the Chevron Fund, and a Department of Education Fellowship (MSJ). We would like to thank D. W. Boo and Prof. Y. T. Lee for their unpublished results.

\section{REFERENCES}

1. T. Y. Yu, T. M. H. Cheng, V. Kempten and F. W. Lampe, J. Phys. Chem. 76, 3321 (1972).

2. J. M. S. Henis, G. W. Stewart, M. K. Tripodi and P. P. Gaspar, J. Chem. Phys. 57, 389 (1972).

3. T. M. H. Cheng, T. Y. Yu and F. W. Lampe, J. Phys. Chem. 78, 1184 (1974).

4. B. H. Boo and P. B. Armentrout, J. Am. Chem. Soc. 109, 3549 (1987).

5. M. L. Mandich, W. D. Reents Jr. and M. F. Jarrold, J. Chem. Phys. 88, 1703 (1988).

6. W. D. Reents Jr. and M. L. Mandich, J. Phys. Chem. 92, 2908 (1988). 
7. M. L. Mandich and W. D. Reents Jr., J. Chem. Phys. 90, 3121 (1989).

8. M. L. Mandich, W. D. Reents and K. D. Kolenbrander, J. Chem. Phys. 92, 437 (1990).

9. W. D. Reents Jr. and M. L. Mandich, J. Chem. Phys. 93, 3270 (1990).

10. K. Raghavachari, J. Chem. Phys. 88, 1688 (1988).

11. K. Raghavachari, J. Phys. Chem. 92,6284 (1988).

12. K. Raghavachari, J. Chem. Phys. 92, 452 (1990).

13. M. A. Al-Laham and K. Raghavachari, J. Chem. Phys. 95, 2560 (1991).

14. G. A. Olah, G. K. S. Prakash, R. E. Williams, L. D. Field and K. Wade, Hypercarbon Chemistry (Wiley-Interscience, New York, 1987).

15. C. H. Hu, M. Shen and H. F. Schaefer III, Chem. Phys. Lett. 190, 543 (1992).

16. J. M. Dyke, N. Jonathan, A. Morris, A. Ridha and M. J. Winter, Chem. Phys. 81, 481 (1983).

17. D. M. Smith, P. M. Martineau and P. B. Davies, J. Chem. Phys. 96, 1741 (1992).

18. M. Okumura, L. I. Yeh and Y. T. Lee, J. Chem. Phys. 88, 79 (1988).

19. L. I. Yeh, M. Okumura, J. D. Myers, J. M. Price and Y. T. Lee, J. Chem. Phys. 91, 7319 (1989).

20. D. W. Boo, J. M. Price and Y. T. Lee, preprint.

21. M. A. Johnson and W. C. Lineberger, in Techniques for the Study of Ion-Molecule Reactions (eds. Farrar, J.M. \& Saunders, W.H., Jr.) 591 (Wiley-Interscience, New York, 1988).

22. D. Proch and T. Trickl, Rev. Sci. Instrum. 60, 713 (1989).

23. S. J. Brosnan and R. L. Byer, IEEE J. Quant. Electron. QE-15, 415 (1979). 